\title{
Editorial
}

\section{ANAESTHESIA IN LAPAROSCOPIC BARIATRIC SURGERY- IS IT SOME THING SPECIAL IN ALL RESPECT ?}

Obesity, derived from the Latin word "Obesus" which means "fattened by eating" has already become a health problem and has reached epidemic proportions in the western countries. The prevalence of obesity in the USA has reached to 19 percent in 1999, and now a days certainly it has been increased to a much higher level.

Obesity is a major health problem with clearly established health implications, including an increased risk of coronary artery disease, hypertension, hyperlipidaemia, diabetes mellitus, gall bladder diseas(;, degenerative joint disease, obstructive sleep apnoea, socioeconomic and psychological impairment. Evidence shows that obesity is associated with increased morbidity and mortality.

"Bariatric", comes from the Greek word "weight treatment" is the only surgery which provides significant and sustained weight loss option for the morbidly obese patients.

For surgery these group of patients are regarded as very high risk patients. Are these patients also fall in high risk group for Anesthesia? These patients are obese and sometimes morbid obese. The Federal guidelines for obesity state three important parameters to assess over weight. (1) Body mass index (BMI), (2) Waist circumference and waist hip ratio, (3) Patients risk factors for diseases associated with Obesity. The world Health organization has endorsed the body mass index as a measure of obesity.

Over weight - BMI $>25-29.9 \mathrm{~kg} / \mathrm{m}^{2}$

Obesity t BMI,> $30-34.9 \mathrm{~kg} / \mathrm{m}^{2}$

Morbid obese- BMI $>35 \mathrm{~kg} / \mathrm{m}^{2}$

The patients who have metabolic syndrome at least three or more of the following criteria must be present

1. Waist circumference $>102 \mathrm{~cm}$ for man and $>$ $85 \mathrm{~cm}$ for woman

2. Serum triglyceride $>100 \mathrm{mg} / \mathrm{dl}$

3. High density lipoprotein $<40 \mathrm{mg} / \mathrm{dl}$ for man and $<50 \mathrm{mg} / \mathrm{dl}$ for woman.
4. Systolic Blood pressure $>130 \mathrm{mmHg}$ or diastolic Blood pressure $>85 \mathrm{mmHg}$

5. tasting blood sugar $>110 \mathrm{mg} / \mathrm{dl}$. /

What procedure the surgeon does in Bariatric Surgery? The surgeon goes for Ma bsorptive or restrictive procedures like vertical banded gastroplasty or gastric banding .

In addition to gross physiological changes in Cardio Vascular Respiratory, Renal and Hepatic, the main changes that occurs in this speciality is the effect of carboperitoneum or venous circulation. Morbid obesity is a major independent risk factor for sudden death from acute postoperative pulmonary embolism.

Increased intra abdominal pressure reverse trendelenburg position high fatty acid level, hyper colesteremia, diabetes accelerated fibrin formation and increased platelet function all promote venous stasis. Increased intralabdominal pressure (IAP) during laparoscopic gastric banding results in reduced peak femoral systolic velocity by $43 \%$ and increase in the femoral cross sectional area by $52 \%$ Nguyea reported that by combining Carboperitoneum to reverse trendelenburg position, peak systolic velocity is decreased to $57 \%$ of base line value.

The sequential compression device (SCD) has been only partially effective in augmenting the femoral peak systolic velocity and the ineffectiveness is attributed to the larger calves and thighs of these individuals.

Special pre-operative evaluation is made regarding CVS specially systemic or pulmonary hypertension, left or right heart failure and Ischemic heart disease. Sites for peripheral or central venous access and for arterial cannulation should be secured before operation.

Another major problem is obstructive sleep apnoea or bronchtic disease. So extreme caution is the rule when administering any pre or postoperative medication that may have a depressant effect on respiratory function. Main goal for several of the 
patient should be a rapid return to a fully alert cooperative state in which the patient can protect the airway. Intensive Care support is a mandatory to maintain the airway either by invasive or non invasive (Bi pap/Cpap) ventilation after operation.

Morbid obese patients of Bariatric Surgery needs induction agents and which are extremely costly. For example propofol is the best and safest induction agents. Likewise desflurane or sevoflurane which are costly and needs special machine as well. Specially designed operation table are required having a maximum weight limit of approximately 205. Special table capable of holding upto $455 \mathrm{~kg}$ with extra width are available but needs huge cost.

In addition to standard monitoring, these group of patients need some special monitoring like Intra abdominal pressure monitoring (IAP), invasive arterial pressure monitoring, and arterial blood gases. In respiratory monitoring needs the information regarding compliance peak and plateau airway pressure, work of breathing etc. which helps for ventilatory setting. Another special monitor which is called bi spectral index monitor to ascertain intra operative awareness is necessary for these patients.

Immediately after operation all most all patients of this group needs ICU support for atlest $24 \mathrm{hrs}$. So to establish this surgical speciality will need adjacent ICU support .

Though bariatric Surgery in morbidly obese patients is associated with higher morbidity and mortality, associated with highest technology needing extraordinary cost, associated with skilled and increased number of man power is still in progress for establishing the field. If special effort is provided, some private sector may come in front and invest huge cogt for developing this specialty. In near future this group of morbidly obese patients will get benefit from the surgery and will be able to maintain more better life.

\section{Dr. Qumrul Huda}

Assistant Professor of Anaesthesia Analgesia \& Intensive Care Medicine, BSMMU, Shahbag, Dhaka-1000. 